\title{
Reply to comment on: Patellar instability in Indian population: relevance of tibial tuberosity and trochlear groove distance
}

\author{
SICOT J 2019, 5, 24, https://doi.org/10.1051/sicotj/2019018
}

\author{
Sourabh Kulkarni, Amith P. Shetty, Karan K. Alva, Saurabh Talekar, and Vijay D. Shetty* \\ Hiranandani Orthopaedic Medical Education (HOME), Dr L. H. Hiranandani Hospital, Hillside Avenue, Hiranandani Gardens, Powai, \\ Mumbai 400076, India
}

Received 5 April 2019, Accepted 11 May 2019, Published online 24 July 2019

To,

The editor

First of all please convey many thanks to author of "Letter to editor" for his interest in our paper [1]. As per the conclusion we have two questions to be answered:

1. Whether the measured parameter should be called as "Patellar tendon-Trochlear Groove distance (PT-TG Distance)" or "Tibial tuberosity-Trochlear Groove Distance (TT-TG Distance)";

2. Methodology used in the study needs to be verified.

It is a very well known fact that any parameter can have more than one method to measure it. Results obtained by one method may differ from another to an extent. But that does not undermine the importance of the actual parameter or does not necessitate to rename it every time when it is measured by a different method. If with each method we change the terminology of that parameter, then confusion is inevitable and importance of the parameter in discussion may fade away.

According to literature, while measuring TT-TG Distance multiple methods are used to define tibial tuberosity and trochlear groove.

To determine tibial tuberosity, the following landmarks are used in various studies:

1. Anterior most point of tibial tuberosity (bony landmark) [2];

2. Center of tibial tubercle (bony landmark);

3. Center of distal most part of patellar tendon attached over tibial tuberosity (cartilaginous/soft tissue landmark) [3, 4].

\footnotetext{
*Corresponding author:vijaydshetty@gmail.com
}

On the trochlear side,

1. The deepest portion of the bony trochlear groove (bony landmark) [2];

2. The deepest portion of the cartilaginous trochlear groove (cartilaginous/ soft tissue landmark).

Also there are multiple studies with contradicting results regarding accuracy of bony vs cartilaginous landmarks and also regarding use of CT scan vs. MR imaging as imaging modalities $[5,6]$.

Even though the landmarks varied in multiple studies, many of them have maintained a discipline to refer measured parameter as TT-TG Distance [3, 4].

We preferred to refer this measurement as TTTG distance considering following points:

1. It is a well-known fact that tibial tuberosity has its significance in discussion because it has patellar tendon attached over it. In other words, tibial tubercle without patellar tendon attachment has no clinical significance and should not be considered for measurement. So we took part of tibial tuberosity for measurement which has patellar tendon attached over it and referred it as "tibial tuberosity (TT)". We did not refer it as "patellar tendon (PT)" because we are measuring the distance from clinically significant tibial tuberosity and not from the patellar tendon.

2. Multiple studies have used the term "TTTG distance" for the same measurement methodology that we used. On the contrary, very few recent articles have come up with newly coined terminology as "PT-TG Distance" for this method. We decided to go by traditional and widely accepted terminology TT-TG distance and not PT-TG distance.

\footnotetext{
This is an Open Access article distributed under the terms of the Creative Commons Attribution License (http://creativecommons.org/licenses/by/4.0), which permits unrestricted use, distribution, and reproduction in any medium, provided the original work is properly cited.
} 
3. In literature we found that two different measurements are being referred as PTTG distance:

(a) patellar tip to trochlear groove distance (PTTG distance) [7];

(b) Patellar tendon to trochlear groove distance (PTTG distance)

So we wanted to avoid confusion.

But we will have no objection to refer this measurement as PTTG distance instead of TTTG distance if

(a) The method used by us to measure the said parameter is unanimously accepted by orthopedic community as PTTG distance for future reference and research;

(b) The confusion about PT-TG distance terminology is resolved.

Now coming to the second question to be answered, there are studies including ours in which the good inter-observer reliability for the measurement has been found. This should resolve the doubt in the methodology.

Hope all the queries are answered to satisfaction.

\section{Conflict of interest}

The authors declare that they have no conflict of interest.

\section{References}

1. Kulkarni S, Shetty AP, Alva KK, Talekar S, Shetty VD (2016) Patellar instability in Indian population: Relevance of tibial tuberosity and trochlear groove distance. SICOT J 2, 14.

2. Goutallier D, Bernageau J, Lecudonnec B (1978) The measurement of the tibial tuberosity. Patella groove distanced technique and results (author's transl). Rev Chir Orthop Reparatrice Appar Mot 64, 423-428.

3. Wittstein J, Bartlett E, Easterbrook J, Byrd J (2006) Magnetic resonance imaging evaluation of patellofemoral malalignment. Arthroscopy 22(6), 643-649.

4. Pandit S, Frampton C, Stoddart J, Lynskey T (2011) Magnetic resonance imaging assessment of tibial tuberosity-trochlear groove distance: normal values for males and females. Int Orthop 35(12), 1799-1803.

5. Wilcox Jason J, Snow Brian J, Aoki Stephen K, Hung Man, Burks Robert T (2012) Does landmark selection affect the reliability of tibial tubercle-trochlear groove measurements using MRI? Clin Orthop Relat Res 470, 2253-2260.

6. Schoettle P, Zanetti M, Seifert B, Pfirmann C, Fucentese S, Romero J (2006) The tibial tuberosity-trochlear groove distance; a comparative study between CT and MRI scanning. Knee 13(1), 26-31.

7. Fischhoff C, Goorah T, Dowlut S. 2015. Ultrasound measurements for patellofemoral pain syndrome: An inter-operator reliability study. Int Musculoskelet Med 37(2), 59-67.

Cite this article as: Kulkarni S, Shetty AP, Alva KK, Talekar S \& Shetty VD (2019) Reply to comment on: Patellar instability in Indian population: relevance of tibial tuberosity and trochlear groove distance. (SICOT J 2019, 5, 24, https://doi.org/10.1051/sicotj/2019018). SICOT-J, 5, 25 